\title{
Adaptive Self-triggered Control over IEEE 802.15.4 Networks
}

\author{
U. Tiberi ${ }^{1}$, C. Fischione ${ }^{2}$, K.H. Johansson ${ }^{2}$ and M.D. Di Benedetto ${ }^{1}$
}

\begin{abstract}
The communication protocol IEEE 802.15.4 is becoming pervasive for low power and low data rate wireless sensor networks (WSNs) applications, including control and automation. Nevertheless, there is not yet any adequate study about control systems networked by this protocol. In this paper, the stability of IEEE 802.15.4 networked control systems (NCSs) is addressed. While in recent works fundamental results are developed for networks that are abstracted only in terms of packet loss and time delays, here the constraints imposed by the protocol to the feedback channel and the network energy consumption are explicitly considered. A general analysis for linear systems with parameter uncertainty and external bounded disturbances with control loops closed over IEEE 802.15.4 networks is proposed. To reduce the number of transmissions and thus save energy, a self-triggered control strategy is used. A sufficient stability condition is given as function of both the protocol and control parameters. A decentralized algorithm to adapt jointly the self-triggered control and the protocol parameters is proposed. It is concluded that stability is not always guaranteed unless protocol parameters are appropriately tuned, and that event-triggered control strategies may be difficult to use with the current version of IEEE 802.15.4.
\end{abstract}

Index Terms-Self-triggered Control, Networked Control Systems (NCSs), IEEE 802.15.4, Wireless Sensor Networks (WSNs).

\section{INTRODUCTION}

Networked Control Systems (NCS) based on energy efficient wireless sensor networks (WSNs) are being widely deployed for many industrial and civilian applications. Building and industrial automation, smart grids, and health care are typical examples [1], [2]. The IEEE 802.15.4 communication protocol is the most popular standard for low power and low data rate WSNs [3]. In industrial automation, it has been adopted with minor variations also by other protocols such as WirelessHART and ISA100 [2]. Moreover, the Internet engineering task force is currently standardizing the RPL routing protocol for WSNs, which is largely based on it [4]. We believe that IEEE 802.15.4 will play a dominant role in WSNs applications as the one played by TCP and UDP protocols for the Internet. Despite such a popularity, there are little or no studies for designing NCSs over IEEE 802.15.4.

In an NCS, sensors take measurements of the plants and send them to the controllers via a communication network. Typical problems are the loss of state information due to

\footnotetext{
1. Department of Electrical and Information Engineering, and Center of Excellence DEWS University of L'Aquila, 67040 Poggio di Roio, L'Aquila, Italy, \{ubaldo.tiberi,mariadomenica.dibenedetto\}@univag. it

2. ACCESS Linnaeus Center, KTH Royal Institute of Technology, Stockholm, Sweden $\{$ carlofi,kallej\} @ee.kth.se

The work of U. Tiberi is partially supported by P.O.R. F.S.E. 2007-2013 Regione Abruzzo, Italy. C. Fischione and K. H. Johansson acknowledge the support of the EU project FeedNetBack, the Swedish Research Council, the Swedish Strategic Research Foundation, and the Swedish Governmental Agency for Innovation Systems.
}

packet dropouts and delays in the packet transmission due to retransmissions, channel contentions among transmitters, and congestions [5]. To cope with these problems, several studies have been proposed in the literature, which we believe can be roughly grouped into three design approaches: a top-down, a bottom up, and a system-level approach, as we discuss next.

By the first approach, packet losses and delays introduced by the communication network are considered as nonidealities, and the controllers are designed without having any influence on them, see e.g., [5] and the references therein. The essential need of energy efficiency usually required by WSNs [2] is often ignored and there is not a systematic attempt to to adapt the protocols so the controllers may work in more favorable conditions. On the other side, protocols for WSNs are often designed to maximize the reliability and minimize the delay to ensure suitable control performance. This is a bottom-up approach, where controller specifications are not explicitly considered even though the protocols are intended to be used for NCSs. This approach is energy inefficient because high reliability and low latency may demand significant energy consumption [2]. A tradeoff between latency, packet losses, and stability requirements can be exploited for the benefit of the energy consumption, as proposed by the system-level design [6]. By following such an approach, entirely new protocol stacks have been developed for control over WSNs, such as Breath and TREnD, see [7], [8].

However, concerning the already standardized protocol as IEEE 802.15.4, there is not yet a systematic study for NCSs. In existing contributions, fundamental results are developed by abstracting the network only in terms of packet losses and time delays, whereas the essential aspect of energy consumption is not considered, and the typical dynamics of network protocols are not taken into account. The idea of adapting communication parameters to the requirements of the controllers is not new, but most of the existing papers are concerned with adaptation of physical layer parameters, including the modulation formats, the radio powers, transmit rates, and Shannon capacity, e.g., [9] - [11], which cannot be adapted by the IEEE 802.15.4 protocol. Some adaptation is possible at the medium access control layer, which however has some strict rules that make it difficult to transmit packets at desired times.

In this paper, we consider the problem of system-level design of NCSs over IEEE 802.15.4 networks. The original contribution is as follows:

1) A self-triggered sampling strategy for ensuring the stability for NCSs where the feedback channel is over an IEEE 802.15.4 network is proposed.

2) A robust stability analysis that takes into account the 
IEEE 802.15.4 protocol parameters is presented.

3) A decentralized algorithm to reduce the energy consumption of the IEEE 802.15.4 network while guaranteeing the stability of the closed loop system is developed.

To the best of our knowledge, this is the first paper that poses the problem of a system-level design of NCSs over IEEE 802.15.4 networks. We focus on self-triggered strategies, because they are energy efficient, as opposed to periodic sampling [12] - [14].

The rest of the paper is organized as follows: in Section II, the IEEE 802.15.4 control system architecture is described and the problem tackled in this paper is introduced. In Section III, an adaptive sampling strategy for IEEE 802.15.4 NCSs is proposed and characterized. In Section IV, numerical simulations illustrate the analysis. Finally, in Section V we summarize the contribution of this paper and future developments.

\section{IEEE 802.15.4 CONTROL System ArChitecture}

We consider a control loop closed on a typical IEEE 802.15.4 star network, where we assume that a node is attached to a plant and transmits state information to a controller that is connected to the network coordinator node.

\section{A. Plant and Controller}

The plant is given by perturbed systems of the form

$$
\dot{x}=A(\eta) x+B(\eta) u+d,
$$

where $x \in \mathbb{R}^{n}, u \in \mathbb{R}^{m}, \eta$ is a vector of parameters uncertainty evolving over a compact set $\mathcal{D}_{\eta} \subset \mathbb{R}^{r}$ and $d \in$ $\mathcal{D}_{d} \in \mathbb{R}^{n}$ is a bounded disturbance with bound $\|d(t)\| \leq \bar{d}$. We assume the following control low:

$$
u(t)=K x\left(t_{k}\right), \quad t \in\left[t_{k}+\tau_{k}, t_{k+1}+\tau_{k+1}\right),
$$

where $t_{k}$ is the time in which the measurements are picked by the sensor attached to the plant, which we call the plant node, and $\tau_{k}$ is the measurement delay, namely the time it takes for the measurement to reach the wireless node attached to the controller, which we call the controller node. This time delay includes the time needed to the processor of the plant node to elaborate the measurement. The control is piecewise constant. Because of the sampling, delays, parameter uncertainties the disturbances, we can obtain only practical stability for the closed loop system.

Definition 2.1: A system $\dot{x}=f(\eta, x, d)$ is locally $\varepsilon$ practically stable if for any $\varepsilon>0$ and for any initial condition $x\left(t_{0}\right) \in \mathcal{D}_{x_{0}} \subseteq \mathbb{R}^{n}$ there exists a time $T \geq 0$ such that $\|x(t)\| \leq \varepsilon$ for all $t \geq t_{0}+T$. If $\mathcal{D}_{x_{0}}=\mathbb{R}^{n}$ then the definition holds globally.

In the following subsection, we see that the times $t_{k}$ and $\tau_{k}$ cannot be imposed only by the controller. They must adapt to the network protocol.

\section{B. Protocol Model}

The IEEE 802.15.4 standard specifies the physical and medium access control layers of the protocol stack of WSNs composed by low cost and low powered nodes [3]. In each

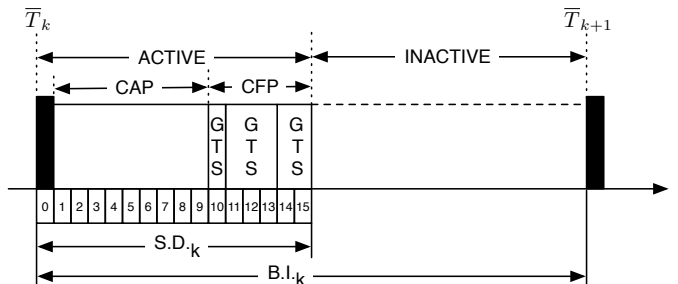

Fig. 1. Slotted IEEE 802.15.4 superframe time organization. The index $k \geq 0$ denotes the $k$ superframe. S.D. $k$ denotes the superframe duration and B.I. ${ }_{k}$ denotes the beacon interval. $\bar{T}_{k}$ is the time in which the superframe begins. A plant node transmits packets during the guarantee time slots (GTS) of the contention free period (CFP). During the inactive period, nodes sleep to save energy. IEEE 802.15 .4 allows us to adapt the protocol parameters S.D. and B.I. to the needs of the NCS.

802.15.4 network there is a special node, the PAN coordinator, that manages the operations of the entire network. We assume that the controller is connected to this coordinator.

The standard allows the network to operate in two different modalities: the unslotted and the slotted one. In the unslotted modality nodes attempt to transmit packets according to the Carrier Sense Multiple Access/Collision Avoidance (CSMA/CA) algorithm, which checks if the channel is idle and randomly back off the transmission if it is busy. In the slotted modality, the nodes transmit packets in a time division multiple access (TDMA) fashion. The time frame of the protocol is denoted as superframe, which is bounded by special signalling packets sent by the PAN coordinator called network beacons to manage the network. Every node of the network must follow this superframe when transmitting packets. The superframe length is denoted as Beacon Interval (B.I.) and satisfies B.I. = aBaseSuperFrameDuration . $\exp (2$, B.O. $)$, with $0 \leq$ B.O. $\leq 14$, where the exponent B.O. is called Beacon Order and aBaseSuperFrameDuration is a parameter of the protocol, which specifies the shortest duration of a superframe.

The superframe is split into an active portion and an inactive portion, see Fig. 1. The active portion is the time interval where there can be transmissions of packets. In the inactive period no communication is allowed and the nodes go in a sleep state to save energy. The time interval of the active period is called Superframe Duration (S.D.). It is divided into 16 equally sized time slots and satisfies

$$
\text { S.D. }=\text { aBaseSuperFrameDuration } \cdot 2^{\text {S.O. }} \text {, }
$$

with $0 \leq$ S.O. $\leq 14$ and where the exponent S.O. is called Superframe Order. It must be S.O. $\leq$ B.O., according to the IEEE standard. The active portion is further divided in two parts: the Contention Access Period (CAP) and the Contention Free Period (CFP). During the CAP nodes contend to access the medium with the CSMA/CA algorithm, whereas in the CFP period the PAN Coordinator reserves dedicated time slots to nodes so that they do not have to contend for transmitting packets during the CFP. During the current superframe, a node can ask to the PAN Coordinator a number of dedicated time slots (up to 7 time slots per superframe). Whenever possible, the PAN Coordinator allocates the required time slots for the next superframe. This mechanism is called Guarantee Time Slots. A time slot is 
called GTS. Note that during a GTS, a node can send and receive more than one packet.

At the beginning of each superframe, all the nodes of the network must be awake to receive the beacon packet from the PAN Coordinator. This beacon packet contains all the settings of the incoming superframe, such as which GTS is reserved to which node, the length of the incoming beacon interval, and the superframe duration. Note that during the inactive period, the nodes are in a sleep state. They wake up to receive the next beacon packet from the PAN Coordinator at the end of the inactive period. The IEEE 802.15.4 standard allows us to adapt the superframe to the need of the controller by tuning the protocol parameters S.D. and B.I., as we state in the following Section.

\section{Problem Formulation}

In an IEEE 802.15.4 NCS, the information about the state of a plant can be sent only during the time intervals of the superframe imposed by the protocol. It follows that one must take into account these constraints imposed by the protocol in the controller design, plus the need of energy efficiency.

Since the nodes of an IEEE 802.15.4 WSN work with batteries with limited charge, a control strategy should minimize the number of transmissions over the network, which consume most of the energy available at the nodes [2]. The protocol parameters (the beacon interval and superframe duration) and the controller should cooperate to put the nodes in a sleep state as long as possible. Then, to reduce both the sampling times and the number of transmissions (thus the network energy consumption), and to obtain $\varepsilon$-practical stability of the closed loop system, we design a self-triggered sampler that has the form

$$
t_{k+1}=t_{k}+\gamma(\zeta)
$$

where $\zeta$ is a vector taking into account the state of the system, time delays etc, and $\gamma(\cdot)$ is a function that we design to obtain 1) $\varepsilon$-practically stability over IEEE 802.15.4 and 2) low network energy consumption. We can state the problem as in the following:

Problem 2.1: Consider the system (1) in which the feedback channel is over an IEEE 802.15.4 network. Determine under which conditions the closed loop system is $\varepsilon$ practically stable by the feedback control (2), and choose the self-triggered sampling instants $t_{k}$ as specified in Eq. (3) that reduces the energy consumption of the network.

In this paper, we assume that nodes transmit over the contention free time slots, the GTSs, as explained above. We consider a static allocation of the GTS in the first slot after the slot of the beacon. We consider the case in which the plant node has one GTS allocated for each supeframe and sends packets during this GTS. Since the beacon packet duration can be at most a slot size, it follows that the measurement of the state of the plant are picked at the time $T_{k} \triangleq \bar{T}_{k}+$ aBaseSuperFrameDuration, where $\bar{T}_{k}$ is the starting time of the $k$-th superframe. By this notation, we remark that $T_{k}$ denotes the sampling time of the plant state allowed by the standard, whereas $t_{k}$ is the sampling time wished by the controller. When the control law is compatible with the standard, then $t_{k} \geq T_{k}$. If $t_{k}<T_{k}$, we may have instability. To allow the plant node to send a packet, the admissible minimum beacon interval is B.I.min $=$ aBaseSuperFrameDuration $\cdot 2$. We assume an initial delay $\tau_{0}=0$ and $\tau_{k} \leq$ aBaseSuperFrameDuration, $\forall k>$ 0 , namely the state information is picked, transmitted, and received inside the GTS time slot. The delay due to the sensing, transmissions is included into the delay $\tau_{k}$. These assumptions are coherent with the IEEE 802.15.4 standard.

\section{SELF-TRIGGERED IEEE 802.15.4 NCSS}

A preliminary result to obtain a robust self-triggered sampler so that we have $\varepsilon$-practical stability of the closed loop system is developed in Section III-A. Then, in Section III-B, we extend this result to an IEEE 802.15.4 network. Finally, in Section III-C, we propose a decentralized algorithm that adapts dynamically the IEEE 802.15.4 protocol parameters to save energy.

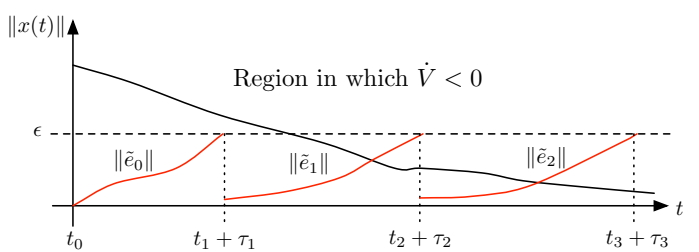

Fig. 2. The proposed self-trigger sampler. The state information $x(t)$ is measured by a plant sensor at time $t_{k}, k=0,1, \ldots$, . The control must be updated at time $t_{k}+\tau_{k}$ as triggered by an error function $\left\|\tilde{e}_{k}\right\|$.

\section{A. A Self Triggered Sampler}

Consider the system (1) with control (2). Once the measurement is taken by the plant node at time $t_{k}$, the closed loop dynamics can be rewritten as

$$
\dot{x}=\left(A_{0}+B_{0} K\right) x+B_{0} K \tilde{e}_{k}+g\left(\eta, x, \tilde{e}_{k}\right)+d,
$$

for $t \geq t_{k}+\tau_{k}$, where $\tilde{e}_{k}(t)=e_{k-1}\left(t_{k}+\tau_{k}\right)+e_{k}(t), e_{k}(t)=$ $x\left(t_{k}\right)-x(t)$ and $A_{0}$ and $B_{0}$ that can be viewed as nominal matrices independent of $\eta$. The assumption $\tau_{0}=0$ implies $\tilde{e}_{0}(t)=e_{0}(t)$. The function $g\left(\eta, x, \tilde{e}_{k}\right)$ in (4) is defined as

$$
\begin{aligned}
g\left(\eta, x, \tilde{e}_{k}\right)= & {\left[\left(A(\eta)-A_{0}\right)+\left(B(\eta)-B_{0}\right) K\right] x+} \\
& +\left(B(\eta)-B_{0}\right) K \tilde{e}_{k},
\end{aligned}
$$

and satisfies $\left\|g\left(\eta, x, \tilde{e}_{k}\right)\right\| \leq\left(\nu_{0}+\nu_{1}\right)\|x\|+\nu_{1}\left\|\tilde{e}_{k}\right\|$, where $\nu_{0} \triangleq \max _{\eta}\left\|A(\eta)-A_{0}\right\|$ and $\nu_{1} \triangleq \max _{\eta}\left\|\left(B(\eta)-B_{0}\right) K\right\|$. To design the self triggered sampler we exploit the behavior of $\left\|e_{k}\right\|$. Note that $\dot{e}_{k}=-\dot{x}$ and, at the sampling time $t_{k}$, $e_{k}\left(t_{k}\right)=0$. Although we don't know exactly $\left\|e_{k}\right\|$, it is possible to find an upper bound for its dynamics by observing that for $t>t_{k}$

$$
\frac{d}{d t}\left\|e_{k}\right\|=\left(e_{k}^{T} e_{k}\right)^{-\frac{1}{2}} e_{k}^{T} \dot{e}_{k} \leq \frac{e_{k}^{T} \dot{e}_{k}}{\left\|e_{k}\right\|} \leq\left\|\dot{e}_{k}\right\|,
$$

and then

$$
\begin{aligned}
\left\|\dot{e}_{k}\right\| \leq & \left\|\left(A_{0}+B_{0} K\right) x\left(t_{k}\right)\right\|+\left(\nu_{0}+\nu_{1}\right)\left\|x\left(t_{k}\right)\right\| \\
& +\left(\left\|A_{0}\right\|+\nu_{0}\right)\left\|e_{k}\right\|+\bar{d} .
\end{aligned}
$$


The previous expression is in the form $\dot{y} \leq a y+b\left\|x\left(t_{k}\right)\right\|+\bar{d}$ where $a \triangleq\left\|A_{0}\right\|+\nu_{0}$ and $b \triangleq\left\|\left(A_{0}+B_{0} K\right)\right\|+\left(\nu_{0}+\nu_{1}\right)$. Because at each sampling $e\left(t_{k}\right)=0$, we have

$$
\left\|e_{k}\right\| \leq \frac{b\left\|x\left(t_{k}\right)\right\|+\bar{d}}{a}\left(e^{a\left(t-t_{k}\right)}-1\right) .
$$

By using this bound, we are now in the position to design the self-triggered sampler that ensures $\varepsilon$-practical stability of the closed loop dynamic (4).

The self-triggered sampler we propose is derived by a Lyapunov approach. We consider the Lyapunov candidate $V(x)=x^{T} P x$, where $P>0$ is solution of the Lyapunov equation $P\left(A_{0}+B_{0} K\right)^{T}+\left(A_{0}+B_{0} K\right) P=-Q<0$ and we define

$$
\begin{gathered}
\xi \triangleq \frac{2 \lambda_{\max }^{P}\left(\left\|B_{0} K\right\|+\nu_{1}+\bar{d}\right)}{\lambda_{\min }^{Q}-2 \lambda_{\max }^{P}\left(\nu_{0}+\nu_{1}\right)}, \\
\varepsilon \triangleq \frac{1}{\xi}\left(\delta-\frac{2 \lambda_{\max }^{P} \bar{d}}{\lambda_{\min }^{Q}-2 \lambda_{\max }^{P}\left(\nu_{0}+\nu_{1}\right)}\right),
\end{gathered}
$$

where $\lambda_{\max }^{M}$ and $\lambda_{\min }^{M}$ denotes the maximum and minimum eigenvalues of a matrix $M$, and $\delta$ is a positive scalar that we choose to determine $\varepsilon$.

Lemma 3.1: Consider the system (1). Let $k_{2}>0$ and $k_{1}>0$ such that $\left\|e^{(A(\eta)+B(\eta) K)\left(t-t_{k}\right)}\right\| \leq k_{1} e^{\alpha\left(t-t_{k}\right)}$ where $\alpha=k_{2}+\nu_{0}+\nu_{1}$. Assume $\alpha<0$ and let a self-triggered sampler be defined as

$$
t_{k+1}=t_{k}+\gamma\left(x\left(t_{k}\right), x\left(t_{k-1}\right), \tau_{k}\right)
$$

with

$$
\begin{aligned}
\gamma\left(x\left(t_{k}\right), x\left(t_{k-1}\right), \tau_{k}\right) & =\frac{1}{a} \ln \left(1+\frac{a \varepsilon}{b\left\|x\left(t_{k}\right)\right\|+\bar{d}}\right. \\
& \left.-\frac{b\left\|x\left(t_{k-1}\right)\right\|+\bar{d}}{b\left\|x\left(t_{k}\right)\right\|+\bar{d}}\left(e^{a \tau_{k}}-1\right)\right)-\tau_{\max } .
\end{aligned}
$$

Then, for a sufficiently large $\delta$ such that

$$
1 \leq \varepsilon\left(\frac{b \max \left\{k_{1}\left\|x\left(t_{0}\right)\right\|, \varepsilon\right\}+\bar{d}}{a}\left(e^{a \tau_{\max }}-1\right)\right)^{-1},
$$

by applying the control (2) at the times given by (7), the closed loop system is local $\varepsilon$-practically stable.

We remark that this lemma requires that $\alpha=k_{2}+$ $\nu_{0}+\nu_{1}<0$, which is only a restriction to the parameter uncertainties. The self-triggered sampler (7) exhibits several interesting properties. Given the measurements $x\left(t_{k}\right)$, $x\left(t_{k-1}\right)$ and the time delay $\tau_{k}$, it allows us to determine the next sampling time $t_{k+1}$ that ensures $\varepsilon$-practical stability. Note that (7) can be used in the cases in which the time delays $\tau_{k}$ are measurable (for example if the packets are time stamped) or not. If the time delays are not measurable, it is always possible to employ the conservative bound $\tau_{k}=\tau_{\max }$. Finally, the proposed self-triggered sampler is robust with respect to parameters uncertainties and external disturbances of the plant and it ensures a non decreasing sequence of times, i.e. $t_{k+1}-t_{k} \geq 0, \forall k$.

However, IEEE 802.15.4 imposes a minimum time interval B.I.min $=$ aBaseSuperFrameDuration $\cdot 2$ between two transmissions, and it must result $t_{k+1}-T_{k} \geq$ B.I.min $_{\min }, \forall k$. This means that a self-triggered sampler that is designed without considering the protocol could give instability. More in general, since event-based sampling have no control of the protocol, it also means that it is very difficult to apply event-based sampling strategies on IEEE 802.15.4 NCS. In the next subsection we investigate the conditions that make self-triggered sampler (7) compatible with IEEE 802.15.4.

\section{B. Stability Condition over IEEE 802.15.4 Networks}

In this subsection, we give one of the core contribution of the paper, namely the system-level design of the controller and the communication protocol.

The self-triggered sampler (7) decreases as both $\left\|x\left(t_{k}\right)\right\|$ and $\tau_{k}$ increase. It depends also on the distance of two consecutive measurements $\left\|x\left(t_{k-1}\right)\right\|$ and $\left\|x\left(t_{k}\right)\right\|$. Since we would like to find a bound on the sampling times of the self-triggered sampler so that it is compatible with IEEE 802.15.4, we begin to find the maximum distance between two consecutive measurements $\left\|x\left(t_{k-1}\right)\right\|$ and $\left\|x\left(t_{k}\right)\right\|$. This distance depends on the size of the $(k-1)$-th beacon interval.

By imposing that the measurements are taken when nodes wakes up to receive the beacon from the PAN coordinator, namely imposing $t_{k}=T_{k}$, we see that the evolution of the system from time $T_{k}$ to time $T_{k+1}$ is bounded with

$$
\begin{aligned}
\left\|x\left(T_{k+1}\right)\right\| \leq & a_{d 1}\left(\text { B.I. } \cdot, \tau_{k}\right)\left\|x\left(T_{k}\right)\right\| \\
& \left.+a_{d 2}\left(\tau_{k}\right)\left\|x\left(T_{k-1}\right)\right\|+a_{d 3} \text { (B.I. } k, \tau_{k}\right),
\end{aligned}
$$

where the terms $a_{d 1}$ (B.I. $\left.{ }_{k}, \tau_{k}\right), a_{d 2}\left(\tau_{k}\right)$ and $a_{d 3}$ (B.I. $\left.{ }_{k}, \tau_{k}\right)$ are derived in [15]. Because the previous functions are increasing with B.I. $k$ and $\tau_{k}$, we can find an upper bound for $\left\|x\left(T_{k+1}\right)\right\|$ given by the solution of the following delay discrete time system:

$$
\left\|x\left(T_{k+1}\right)\right\|=A_{d 1}\left\|x\left(T_{k}\right)\right\|+A_{d 2}\left\|x\left(T_{k-1}\right)\right\|+A_{d 3},
$$

where $A_{d 1}=a_{d 1}$ (B.I. max $\left._{\max }, \tau_{\max }\right), A_{d 2}=a_{d 2}\left(\tau_{\max }\right)$ and $A_{d 3}=a_{d 3}$ (B.I.max,$\left.\tau_{\max }\right)$. We have the following $\varepsilon$-practical stability sufficient condition over IEEE 802.15.4 NCSs:

Theorem 3.1: Consider the system (1) and let the assumptions of Lemma 3.1 hold. Suppose there exists a B.I.min such that

$$
\frac{1}{a} \ln \left(1+\frac{\varepsilon}{\rho_{1}}-\frac{\rho_{0}}{\rho_{1}}\left(e^{a \tau_{\max }}-1\right)\right)-\tau_{\max } \geq \text { B.I. } \min ,
$$

where $\rho_{0}=a^{-1}\left(b \max \left\{\kappa_{1}\left\|x\left(t_{0}\right)\right\|, \varepsilon\right\}+\bar{d}\right)$, and $\rho_{1}=$ $a^{-1}\left(b\left(\left(A_{d 1}+A_{d 2}\right) \max \left\{\kappa_{1}\left\|x\left(t_{0}\right)\right\|, \varepsilon\right\}+A_{d 3}\right)+\bar{d}\right)$. Then using the control (2) and the self-triggered sampler (7), the system is local $\varepsilon$-practically stable over an IEEE 802.15.4 network.

We remark that Theorem 3.1 captures a tradeoff among the admissible set of initial conditions $x_{0}$, the maximum delay allowed $\tau_{\max }$ to transmit plant measurements, the maximum B.I. allowed, the sets of the parameters uncertainties $\mathcal{D}_{\eta}$ and the external perturbations $\mathcal{D}_{d}$, and the time constants of both the open and the closed loop systems. Hence, in the design of an IEEE 802.15.4 NCS, if Theorem 3.1 applies, then the closed loop system is $\varepsilon$-practically stable and the self-triggered sampler (7) can be successfully used. 
Because the self-triggered sampler gives larger times as the norm of the state decreases, it could happen that $t_{k+1}-$ $T_{k}>>$ B.I. $k$ for some $k$ when the norm is sufficiently small. We can increase the beacon interval to reduce the number of transmissions and to increase the inactive periods. In the next section we propose a distributed algorithm to achieve this goal.

\section{Adaptive IEEE 802.15.4}

In this subsection, we show how the IEEE 802.15.4 MAC parameters can be adapted so that the self-triggered sampler ensures stability and the energy consumption of the network is reduced. In particular, here we deal with three issues

1) The adaptation of the MAC parameters, namely the duration of the beacon interval, must be coordinated between the plant node and the controller node.

2) The sampling times given by the self-triggered sampler

(7) must be larger than the minimum beacon interval allowed by the IEEE 802.15.4 standard.

3) The sampling times given by the self-triggered sampler (7) must be lower than the maximum beacon interval allowed by the IEEE 802.15.4 standard.

In the following, we investigate these issues and propose Algorithm 1, which summarizes the adaptation mechanism of the IEEE 802.15.4 MAC parameters to the self-triggered strategy.

The times $t_{k}$ given by the self-triggered sampler (7) are hard deadlines by which the state measurements must be taken and transmitted. Theorem 3.1 guarantees $T_{k}<t_{k+1}<$ $T_{k+1}, \forall k$. However, at time $t_{k}+\tau_{k}$, i.e. when $t_{k+1}$ is computed, it could happen that $T_{k}<t_{k}<T_{k+1}<T_{k+2}<$ $\cdots<T_{k+m}<t_{k+1}$. This means that the nodes may wake up and transmit data even if it is not needed. It is then reasonable to provide an increasing of the $k$-th beacon interval, so that $T_{k+1}<t_{k+1}<T_{k+2}, \forall k$. An increasing of the beacon interval gives two benefits: a reduction of the number of transmissions, and a reduction of the duty cycle of the $(k+1)$-th superframe, thus reducing the energy consumption of the network.

As described in II-B, the standard doesn't allow us to change the $k$-th superframe parameters at time $t$ with $T_{k}<$ $t<T_{k+1}$. However, at a time $t$ it is possible to decide the structure of the $(k+1)$-th superframe and encapsulate this information in the next beacon packet. To enlarge the $(k+1)$-th beacon interval, we use the estimate $\left\|\hat{x}\left(T_{k+1}\right)\right\|$ of $\left\|\hat{x}\left(T_{k+1}\right)\right\|$, that can be computed by (10), and we use the self-triggered sampler (7) with

$$
\hat{t}_{k+2}=T_{k+1}+\gamma\left(\hat{x}\left(T_{k+1}\right), x\left(T_{k}\right), \tau_{k}\right) .
$$

If $T_{k+2}<\hat{t}_{k+2}$ it is then possible to increase the $(k+1)$-th beacon interval as we show below by Proposition 3.1.

On the other hand, a beacon interval computed by the self-triggered strategy could be too large. By using the same arguments as above, we can reduce the $(k+1)$-th beacon interval if $\hat{t}_{k+2}<T_{k+2}$. It is worth noting that by reducing the beacon interval to B.I. min $_{\text {, }}$ the self-triggered sampler continues to work correctly as per Theorem 3.1.

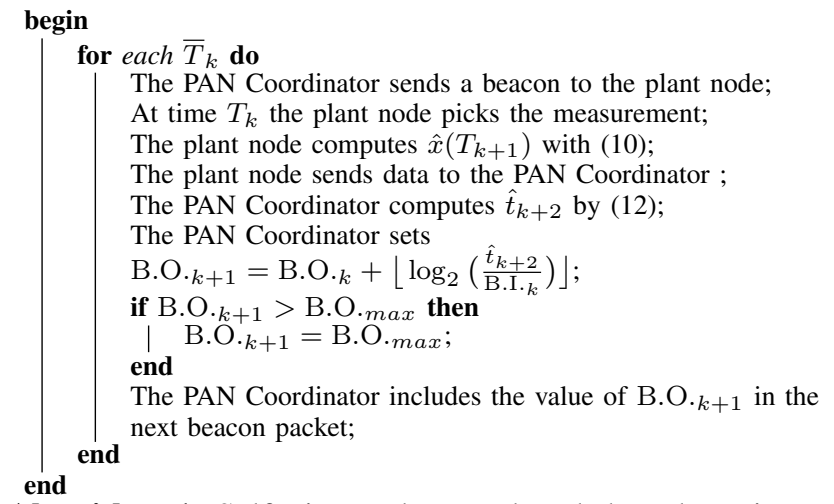

Algorithm 1: Self-triggered control and the adaptation of IEEE 802.15.4 MAC.

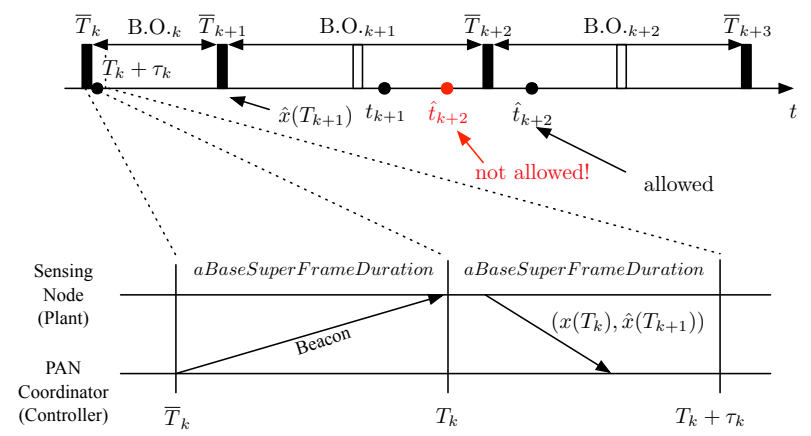

Fig. 3. Adaptive IEEE 802.15.4 Algorithm: a) The PAN Coordinator sends the beacon to the plant node at time $\bar{T}_{k}$. b) The plant node picks the state measurement at time $T_{k} x\left(T_{k}\right)$, estimates $\hat{x}\left(T_{k+1}\right)$ and sends back these data to the PAN Coordinator. c) The coordinator computes $\hat{t}_{k+2}$ and B.O. ${ }_{k+1}$ and encapsulates this value in the next beacon packet, so that the plant node receives the beacon at time $\bar{T}_{k+1}$ and adapts its beacon interval accordingly.

A sketch of the algorithm is depicted in Fig. 3 while Algorithm 1 gives a formal explanation. Note that Algorithm 1 gives an estimated time $\hat{t}_{k+2}$ such that $T_{k+2}<\hat{t}_{k+2}$. Since it must be also that $T_{k+2}<t_{k+2}$, this is ensured by the following result:

Proposition 3.1: If the hypothesis of Theorem 3.1 hold, then Algorithm 1 ensures that $t_{k}<T_{k+1}<t_{k+1}, \forall k$. $\triangleleft$

Algorithm 1 respects the IEEE 802.15.4 protocol constraints while ensuring stability. Such algorithm has many benefits in terms of energy consumption and implementation aspects. First, in contrast to a periodic sampling time with, e.g., B.I.min, which would give much worse energy consumption, it increases the beacon interval. Second, the computations can be distributed to the nodes of the network: the state estimator (10) can be implemented at the plant node, while the self-triggered sampler (12) can be implemented at the controller. The plant node transmits the values of $x\left(T_{k}\right)$ and $\hat{x}\left(T_{k+1}\right)$ while the PAN Coordinator updates the control law, computes $\hat{t}_{k+2}$, and encapsulates the value of B. $\mathrm{O}_{k+1}$ in the $(k+1)$-th beacon, see Fig. 3 .

\section{Simulations}

In this section, we report some simulation results that illustrate the proposed self-triggered strategy for IEEE 802.15.4 NCSs. We simulated the scenario of Section II and imple- 
mented Algorithm 1. We considered system (1) with

$$
A(\eta)=\left[\begin{array}{cc}
-0.2+\eta_{1} & 1 \\
0 & 0.05+\eta_{2}
\end{array}\right], B(\eta)=\left[\begin{array}{c}
1+\eta_{3} \\
2
\end{array}\right] .
$$

In this example we used the bound on the matrix exponential given by $\left\|e^{A t}\right\| \leq k_{1} e^{k_{2} t}$ with $k_{1}=1$ and $k_{2}=$ $0.5 \lambda_{\max }^{\left(A_{0}+B_{0} K\right)+\left(A_{0}+B_{0} K\right)^{T}}$, as proposed in [16].

The parameters uncertainty $\eta_{1}, \eta_{2}$ and $\eta_{3}$ evolve randomly in the set $[-0.01,0.01]$ during the simulations, and the maximum value of the external disturbance is set to $\bar{d}=0.02$. We designed a control $u=K x$ that places the closed loop nominal system eigenvalues at $\lambda_{1}=-0.2$ and $\lambda_{2}=-0.1$. We set $x_{1}(0)=100, x_{2}(0)=70$ as initial conditions. For aBaseSuperFrameDuration $=4 \mathrm{~ms}$, a maximum delay $\tau_{\max }=0.2 \mathrm{~ms}$, and B.O. $\cdot_{\max }=11$, the stability condition (11) provided a minimum inter sampling time of $\simeq 8.3 \mathrm{~ms}>$ B.I.min $=$ aBaseSuperFrameDuration $\cdot 2=8 \mathrm{~ms}$. Thus, the condition (11) is verified and the closed loop system is $\varepsilon$-practically stable over the network.

In Fig. 4 we report the behavior of the system in the cases when we apply the designed continuous control $u_{c}(t)=K x(t)$ and the piecewise constant control $u(t)=$ $K x\left(T_{k}\right), t \in\left[T_{k}+\tau_{k}, T_{k+1}+\tau_{k+1}\right)$. The system results $\varepsilon-$ practically stable for both controls.

In Fig. 5 we reported the adaptation of the beacon order $\mathrm{B} \mathrm{O}_{k}$ as determined by Algorithm 1. Note how the plant node sent measurements over longer time intervals as the state norm decreases.

We conclude by noting that Algorithm 1 achieved stability, reduced the number of transmission, and increased the inactive periods of the beacon intervals. A reduction of the network energy consumption was achieved as a consequence.

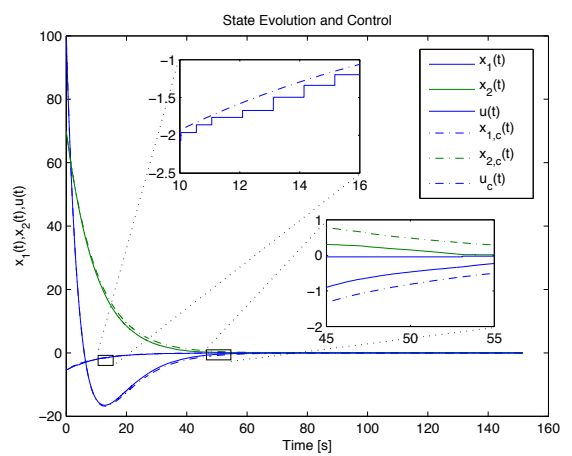

Fig. 4. System response and control. The dotted lines denote the system response and the control as obtained by a continuous control $u_{c}(t)=$ $K x(t)$, and the continuous lines denote the evolution of the state and the control obtained by our Algorithm 1.

\section{CONClusions And Future Work}

We presented an analysis for networked control systems when the feedback channel is closed over an IEEE 802.15.4 network. A sufficient condition for the stability over such networks was derived. An algorithm that provides a dynamic adaptation of the protocol parameters to obtain energy saving and stability was proposed.

Future works include the investigation of the fundamental stability conditions for more complex network topologies,

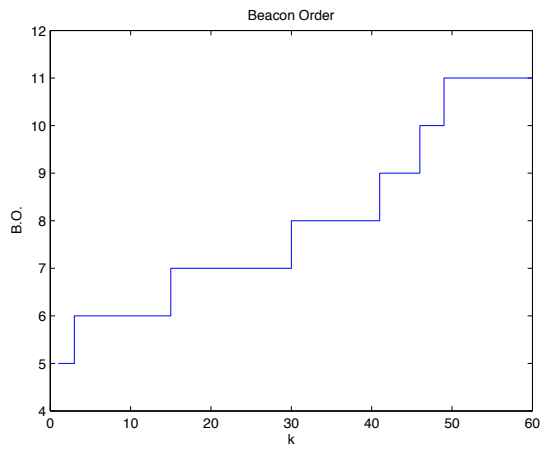

Fig. 5. IEEE 802.15 .4 beacon order adaptation as obtained by our Algorithm 1. On the $\mathrm{x}$-axis, the superframe number is reported.

possibly with the multihop routing of the RPL protocol [4]. We also plan to include systems composed by more plants and controllers that share the same IEEE 802.15.4 network. The problem of scheduling the guaranteed time slots, for each combination of number of sensors and controllers, is under development.

\section{REFERENCES}

[1] N. J. Ploplys, P. A. Kawka, and A. G. Alleyne, "Closed-loop control over wireless networks," IEEE Control Systems Magazine, vol. 24, no. 3, pp. 58-71, 2004.

[2] A. Willig, "Recent and emerging topics in wireless industrial communication," IEEE Transactions on Industrial Informatics, vol. 4, no. 2 , pp. 102-124, 2008.

[3] Wireless Medium Access Control (MAC) and Physical Layer (PHY) Specifications for Low Rate Wireless Personal Area Networks (LR-WPANs), IEEE 802.15.4, 2006. [Online]. Available: http://standards.ieee.org/getieee802/download/802.15.4-2006.pdf

[4] Routing Over Low power and Lossy networks (roll). [Online]. Available: http://www.ietf.org/dyn/wg/charter/roll-charter.html

[5] J. P. Hespanha, P. Naghshtabrizi, and Y. Xu, "A survey of recent results in networked control systems," Proceedings of the IEEE, vol. 95, no. 1, January 2007.

[6] A. Bonivento, C. Fischione, L. Necchi, F. Pianegiani, A. SangiovanniVincentelli, "System level design for clustered wireless sensor networks," IEEE Transactions on Industrial Informatics, pp. 202-204, 2007.

[7] P. Park, "Protocol design for control applications using wireless sensor networks," Royal Institute of Technology (KTH), Tech. Rep. TRITAEE 2009:041, Oct. 2009, licentiate Thesis.

[8] P. D. Marco, P. Park, C. Fischione, and K. H. Johansson, "TREnD: a timely, reliable, energy-efficient and dynamic wsn protocol for control applications," in Proceedings of the Information Communicatoin Conference, June 2010.

[9] L. Xiao, M. Johansson, H. Hindi, S. Boyd, and A. Goldsmith, "Joint optimization of communication rates and linear systems," IEEE Transactions on Automatic Control, vol. 48, no. 1, pp. 148-153, 2003.

[10] B. Azimi-Sadjadi, "Stability of networked control systems in the presence of packet losses," in Proceedings of the 41st Conference on Decision and Control, December 2003.

[11] L. Bao, "Control over low-rate noisy channels," Ph.D. dissertation, Royal Institute of Technology (KTH), Jun. 2009, tRITA-EE 2009:032.

[12] M. Velasco, P. Marti, and J. Fuertes, "The self triggered task model for real-time control systems," in Work-in-Progress Session of the $24^{\text {th }}$ IEEE Real-Time Systems Symposium (RTSSO3), 2003.

[13] M. Mazo and P. Tabuada, "On event-triggered and self-triggered control over sensor/actuator networks," in $47^{\text {th }}$ IEEE Conference on Decision and Control, December 2008.

[14] X. Wang and M. Lemmon, "Self-triggered feedback control systems with finite-gain $\mathcal{L}_{2}$ stability," IEEE Transactions on Automatic Control, vol. 54, no. 3, pp. 452-467, March 2009.

[15] U. Tiberi, C. Fischione, K. H. Johansson, and M. D. Benedetto, "Adaptive self triggered control over 802.15.4 networks," Royal Institute of Technology, Sweden, Tech. Rep., 2010.

[16] B. Kågström, "Bound and perturbation bounds for the matrix exponential," in BIT Numerical Mathematics, 1976, pp. 39-57. 